NISSUNA UMANA INVESTIGAZIONE SI PUO DIMANDARE VERA SCIENZIA S'ESSA NON PASSA PER LE MATEMATICHE DIMOSTRAZIONI LEONARDO DA VINCI

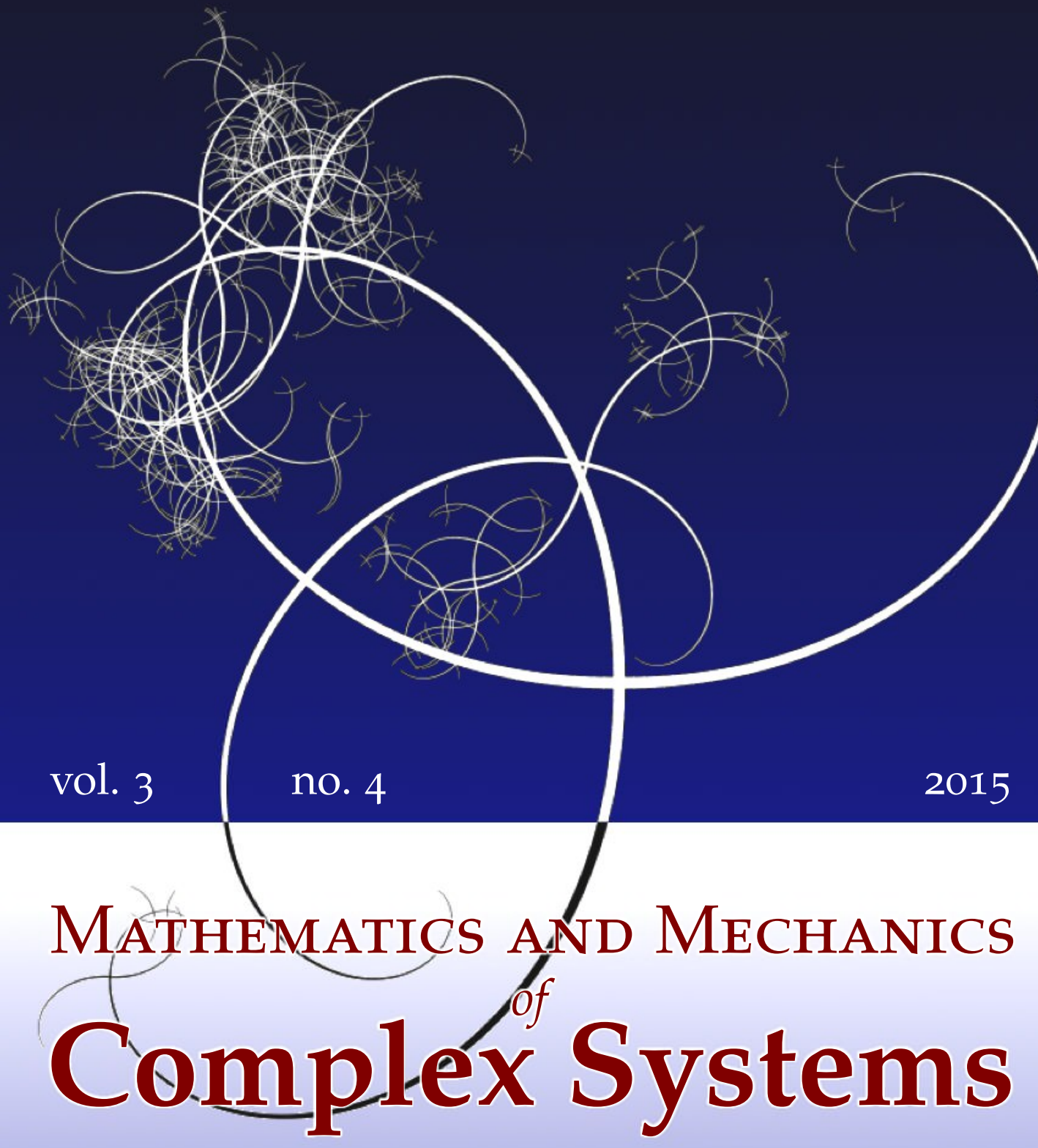

JEAN-FrANÇOIS GANGHOFFER

SPATIAL AND MATERIAL STRESS TENSORS IN CONTINUUM MECHANICS OF GROWING SOLID BODIES 


\title{
SPATIAL AND MATERIAL STRESS TENSORS IN CONTINUUM MECHANICS OF GROWING SOLID BODIES
}

\author{
JEAN-FRANÇOIS GANGHOFFER
}

\begin{abstract}
We presently derive generalized expressions of the stress tensor for continuum bodies with varying mass, considering both the Lagrangian and Eulerian viewpoints in continuum mechanics. We base our analysis and derivation of the expressions of both Cauchy and Eshelby stress tensors on an extension of the virial theorem for both discrete and continuous systems of material points with variable mass. The proposed framework is applicable to describe physical systems at very different scales, from the evolution of a population of biological cells accounting for growth to mass ejection phenomena occurring within a collection of gravitating objects at the very large astrophysical scales. As a starting basis, the field equations in continuum mechanics are written to account for a mass source and a mass flux, leading to a formulation of the virial theorem accounting for a varying mass within the considered system. The scalar and tensorial forms of the virial theorem are written successively in both Lagrangian and Eulerian formats, incorporating the mass flux. This delivers generalized formal expressions of Cauchy and Eshelby stress tensors versus the average tensor spatial and material virials respectively, incorporating the mass flux contribution.
\end{abstract}

\section{Introduction}

There are many problems in physics which involve masses changing with time, as exemplified by situation of growing bodies, solids and fluids exhibiting phase transitions [Ericksen 1984] related to solidification, evaporation, sedimentation. In particular, the mass balance (mass absorbency) influence phase transitions conditions, see for instance [dell'Isola and Iannece 1989; Eremeyev and Pietraszkiewicz 2009; 2011]. To mention but a few, two specific situations illustrate the very wide range of scales at which such phenomena may occur: growth or resorption in biological systems is a typical situation where the overall mass of a continuous body or a collection of particles varies, due to mass production within the system, or to a flux of mass through the system boundary. Growth at cellular level (individual

\section{Communicated by Francesco dell'Isola.}

MSC2010: primary 74B20; secondary 70FXX.

Keywords: continuum bodies with changing mass, generalized virial theorem, discrete mechanics, mass flux, Eshelby stress. 
cells can then be regarded as punctual masses) is typical of discrete growth, while the continuous aspect is of relevance for large collections of cells, organized within tissues for which the framework of continuous mechanics is adequate.

At a much larger scale, the dynamics of galaxies with mass loss due to either mass accretion or mass ablation has deserved a lot of attention in the literature for many years, usually relying on an extension of Newton's law of motion [Gommerstadt 2001], as originally stated by Sommerfeld in 1952 .

In 1870, Rudolf Clausius [1870] stated that the mean vis viva of the system is equal to its virial, or that the average kinetic energy is equal to half the average potential energy. The virial theorem which was there born is a way to analyze the dynamics of a collection of interacting particles; it allows the average total kinetic energy to be calculated from the potential energy of a stable system. This holds even for very complicated systems that defy an exact solution, such as those considered in statistical mechanics or in astrophysics when considering large scales. Lord Rayleigh published a generalization of the virial theorem in 1903. Henri Poincaré applied a form of the virial theorem to the problem of determining the cosmological stability in 1911. A tensor form of the virial theorem was set up in [Chandrasekhar and Fermi 1953; Chandrasekhar and Lebovitz 1962; Parker 1954], both in the context of astrophysics.

The virial theorem has a rather broad physical significance; it has been extended to include electric and magnetic fields. The virial has both a discrete and a continuum facet, the first facet being well adapted to the physical situation of a finite collection of particles, while the continuum virial obtained by some kind of averaging process brings a simplification by introducing fields in place of discrete quantities. In the context of continuum mechanics, the virial theorem proves an alternative efficient manner to derive the pressure for particles without internal structure (fluids), avoiding thereby the - sometimes complex - derivation of a thermodynamic potential (the free energy).

The virial theorem has raised a renewed interest in the contemporary literature in relation to the construction of the Cauchy stress for structured media, derived from the tensorial virial theorem in [Jouanna and Brocas 2001; Jouanna and Pèdesseau 2004], borrowing arguments from statistical mechanics. The general idea at the root of the molecular definition of the average stress is the identification of molecules or atoms as interacting point masses. This reminds to the similar pioneering work of Irving and Kirkwood [1950], in which stress is defined as a pointwise statistical averaging performed in time instead of space, relying on the ergodicity hypothesis. This viewpoint applies for a number of molecules which is large enough for averaging operations to make sense - instead of using quantum mechanics - so that a classical description can be adopted. Works in the literature since this pioneering contribution witness a diversity of definitions and derivations 
of the stress tensor using a molecular viewpoint, see the recent critical overview [Murdoch 2007] and references therein. Especially, when micro-macro identifications processes are considered, higher gradient theories naturally arise in which to Cauchy stress tensor one needs to add a family of hyper stresses, as already remarked by Gabrio Piola in his pioneering works [dell'Isola and Iannece 1989; dell'Isola et al. 2012; 2014].

As a main outcome and novelty of the present contribution, one shall derive expressions of the material Eshelby stress and spatial Cauchy stress tensors for continuum bodies witnessing a local change of their mass. We base our analysis and derivation of the expressions of both Cauchy and Eshelby stress tensors on an extension of the virial theorem for both discrete and continuous systems of material points with variable mass, thereby generalizing developments exposed in [Ganghoffer 2010b]. Eshelby stress appears as a driving force for the growth of continuum solid bodies, possibly incorporating multiphysical phenomena, see [Ganghoffer 2010a; 2012].

The present contribution is organized as follows. In order to set the stage, the virial theorem is first recalled in both scalar and tensorial formats (Section 2). We next extend in Section 3 the virial theorem for systems with variable mass, a situation which occurs for growing biological systems and for a set of gravitational masses with mass loss at the other extreme of the length spectrum. The virial theorem for systems with variable mass is derived in sections 2 and 3 in Eulerian format, and the material counterpart is written in sections 4 and 5, highlighting the variation of the average virial in relation to the divergence of Eshelby stress. A summary of the main developments is given in Section 6.

A few words regarding notation are in order. Vectors and higher-order tensors are denoted with boldface symbols. Likewise, tensorial quantities built from their scalar counterparts are denoted as boldface characters with a superposed hat; e.g., $\hat{\boldsymbol{E}}_{k}$ denotes the tensorial kinetic energy, such that its trace is the scalar kinetic energy: $\operatorname{Tr}\left(\hat{\boldsymbol{E}}_{k}\right)=E_{k}$. The summation convention on repeated indices is in force, unless otherwise explicitly stated.

The bracket $\langle\cdot\rangle$ denotes the ensemble average of any quantity. The partial derivative of a scalar function $f(x)$ is denoted $\partial_{x} f=\partial f / \partial x$; the time derivative of a function $\boldsymbol{a}(t)$ is represented by a superposed dot: $\dot{\boldsymbol{a}}(t)=d \boldsymbol{a}(t) / d t$. The material and spatial gradients are denoted Grad $\equiv \nabla_{R}$ and grad $\equiv \nabla$ respectively; similarly, the material and spatial divergence are $\operatorname{denoted} \operatorname{Div}(\cdot) \equiv \nabla_{R}(\cdot)$ and $\operatorname{div}(\cdot)=\nabla \cdot(\cdot)$ respectively. The transpose of the linear mapping $A$ is the linear mapping denoted $A^{T}$. The notation $\operatorname{sym}(\cdot)$ stands for the symmetrized part of a dyadic product. The second-order identity tensor is denoted $\boldsymbol{I}$. 


\section{Nomenclature of the principal symbols}

\begin{tabular}{|c|c|}
\hline $\boldsymbol{R}, \boldsymbol{R}_{i}\left(\boldsymbol{r}, \boldsymbol{r}_{i}\right)$ & material (resp. spatial) position vectors \\
\hline$J$ & inertia tensor \\
\hline $\boldsymbol{p}_{i}:=m_{i} \dot{\boldsymbol{r}}_{i}$ & momentum of a single particle \\
\hline$\hat{V}, \hat{V}$ & $\begin{array}{l}\text { scalar and tensorial virials ; } \hat{V}_{\text {int }}, \hat{V}_{\text {ext }} \text { internal } \\
\text { and external scalar virials }\end{array}$ \\
\hline$\langle\cdot\rangle$ & $\begin{array}{l}\text { ensemble averaging (equivalent to time averaging } \\
\text { according to ergodicity) }\end{array}$ \\
\hline$\left\langle\hat{V}_{0}\right\rangle\left(\operatorname{resp} .\left\langle\hat{\boldsymbol{V}}_{0}\right\rangle\right)$ & average scalar (resp. tensorial) material virial \\
\hline$\langle\hat{V}\rangle($ resp. $\langle\hat{\boldsymbol{V}}\rangle)$ & average scalar (resp. tensorial) spatial virial \\
\hline$E_{k}, E_{p}$ & kinetic and potential energy respectively \\
\hline$\hat{\boldsymbol{E}}_{k}, \hat{\boldsymbol{E}}_{p}$ & tensorial kinetic and potential energy respectively \\
\hline $\boldsymbol{F}:=\operatorname{Grad} \boldsymbol{r}$ & first-order transformation gradient \\
\hline \multicolumn{2}{|l|}{$\boldsymbol{J}:=\operatorname{det}(\boldsymbol{F})$} \\
\hline$\psi$ & kinematically admissible position field \\
\hline$f_{0}, f$ & referential and spatial body forces \\
\hline${ }^{3} \boldsymbol{G}:=\nabla_{X} \boldsymbol{F}$ & second-order transformation gradient \\
\hline$W_{0}(\boldsymbol{F})$ & strain energy density \\
\hline $\boldsymbol{T}:=\partial W_{0}(\boldsymbol{F}) / \partial \boldsymbol{F}$ & first Piola-Kirchhoff stress \\
\hline$\sigma$ & Cauchy stress tensor \\
\hline$\underset{\sim}{\boldsymbol{\Sigma}}:=W_{0} \boldsymbol{I}-\boldsymbol{F}^{t} \cdot \boldsymbol{T}$ & second-order Eshelby stress \\
\hline$\tilde{\tilde{\boldsymbol{\Sigma}}}:=W_{0} \boldsymbol{I}^{4}-\boldsymbol{F}^{t} \otimes \boldsymbol{T}$ & fourth-order Eshelby stress \\
\hline
\end{tabular}

\section{Scalar and tensorial virial theorems for systems with constant mass}

In order to set the stage, a reminder of the statement of the virial theorems in both scalar and tensorial format for systems of particles with constant mass are conveniently recalled.

The Lagrangian of a set of $N$ point particles with mass $m_{i}$ and position vector $\boldsymbol{r}_{i}$, moving in a potential $E_{p}\left(\left\{\boldsymbol{r}_{i}\right\}\right)$, is the difference between the kinetic energy

$$
E_{k}\left(\left\{\dot{\boldsymbol{r}}_{i}\right\}\right):=\sum_{i=1}^{N} \frac{\boldsymbol{p}_{i}^{2}}{2 m_{i}},
$$

where $\boldsymbol{p}_{i}:=m_{i} \dot{\boldsymbol{r}}_{i}$ is the momentum of the $i$-th particle, and the potential energy:

$$
L\left(\left\{\boldsymbol{r}_{i}, \dot{\boldsymbol{r}}_{i}\right\}\right):=E_{k}\left(\left\{\dot{\boldsymbol{r}}_{i}\right\}\right)-E_{p}\left(\left\{\boldsymbol{r}_{i}\right\}\right) .
$$

In the hamiltonian formulation, the independent variables are the spatial positions and the momenta, namely the set of variables $\left\{\boldsymbol{r}_{i}, \boldsymbol{p}_{i}\right\}$. The Lagrangian is related 
to the Hamiltonian

$$
H\left(\left\{\boldsymbol{r}_{i}, \boldsymbol{p}_{i}\right\}\right):=E_{k}+E_{p},
$$

defined as the sum of the kinetic and potential energies of the individual particles, by the equation

$$
L(\boldsymbol{r}, \dot{\boldsymbol{r}} ; t)=\boldsymbol{p} \cdot \frac{d \boldsymbol{r}}{d t}-H(\boldsymbol{r}, \boldsymbol{p} ; t) .
$$

Each material point is submitted to a force given by the gradient of the potential energy vs. the corresponding spatial position, hence

$$
f_{i}=-\partial_{r_{i}} E_{p} .
$$

The scalar virial theorem states that the virial, viz the scalar valued quantity

$$
\hat{V}:=\sum_{i=1}^{N} \boldsymbol{r}_{i} \cdot \boldsymbol{f}_{i}
$$

is related to the kinetic energy of the set of particles by (the arguments of the functionals are omitted for the sake of simplicity)

$$
\frac{d}{d t}\left(\sum_{i=1}^{N} \boldsymbol{p}_{i} \cdot \boldsymbol{r}_{i}\right)=2 E_{k}+\hat{V} .
$$

In the asymptotic limit of infinite times, the time average - indicated by the bracket operator - of the left side of the previous identity vanishes, hence the ensemble average of the right side vanishes:

$$
2\left\langle E_{k}\right\rangle+\langle\hat{V}\rangle=0 .
$$

This identity constitutes the scalar version of the virial theorem. The assumption of ergodicity at the macroscopic equilibrium implies that time averages at fixed coordinate (following a single particle) are interchangeable with ensemble averages (averages over a sufficiently large set of particles) at fixed time.

The virial can be decomposed into the sum of the internal virial $\hat{V}_{\text {int }}$ and the external virial $\hat{V}_{\text {ext }}$, as

$$
\hat{V}=\left(\boldsymbol{f}_{i j} \cdot \boldsymbol{r}_{i j}\right)_{j \neq i}+\boldsymbol{f}_{i, \mathrm{ext}} \cdot \boldsymbol{r}_{i} \equiv \hat{V}_{\mathrm{int}}+\hat{V}_{\mathrm{ext}}
$$

highlighting the contribution of internal forces $f_{i j}$ (first term on the right due to interparticle interactions) and external forces (body forces due to gravity and contact), denoted $\boldsymbol{f}_{i \text {, ext }}$, adopting the notation $\boldsymbol{r}_{i j}:=\boldsymbol{r}_{i}-\boldsymbol{r}_{j}$ for the relative position of particles $i$ and $j$.

The generalized virial theorem established in [Jouanna and Brocas 2001], viz the tensorial generalization of the identities (2-6), (2-7), (2-8), can be obtained as 
follows: let differentiate twice the (symmetrical) inertia tensor (the summation of repeated indices in the dyadic products is done over the set of $N$ particles)

$$
\boldsymbol{I}:=m_{i} \boldsymbol{r}_{i} \otimes \boldsymbol{r}_{i}
$$

hence giving

$$
\frac{d \boldsymbol{I}}{d t}:=m_{i}\left(\dot{\boldsymbol{r}}_{i} \otimes \boldsymbol{r}_{i}+\boldsymbol{r}_{i} \otimes \dot{\boldsymbol{r}}_{i}\right)
$$

and so

$$
\frac{d^{2} \boldsymbol{I}}{d t^{2}}=2 m_{i} \dot{\boldsymbol{r}}_{i} \otimes \dot{\boldsymbol{r}}_{i}+m_{i}\left(\ddot{\boldsymbol{r}}_{i} \otimes \boldsymbol{r}_{i}+\boldsymbol{r}_{i} \otimes \ddot{\boldsymbol{r}}_{i}\right)=2 \hat{\boldsymbol{E}}_{k}+\hat{\boldsymbol{V}}
$$

with

$$
\hat{\boldsymbol{V}}:=2 \operatorname{sym}\left(\boldsymbol{r}_{i} \otimes \boldsymbol{f}_{i}\right)=-2 \operatorname{sym}\left(\boldsymbol{r}_{i} \otimes \partial_{r_{i}} E_{p}\right)
$$

defined as the tensorial virial, and the tensorial kinetic energy elaborated as

$$
\hat{\boldsymbol{E}}_{k}:=2 m_{i} \dot{\boldsymbol{r}}_{i} \otimes \dot{\boldsymbol{r}}_{i} \equiv \frac{\boldsymbol{p}_{i} \otimes \boldsymbol{p}_{i}}{2 m_{i}} .
$$

Remark. The trace of $\hat{\boldsymbol{V}}$ gives the scalar virial, $\operatorname{Tr}(\hat{\boldsymbol{V}})=\hat{V}$; similarly, the scalar kinetic energy is recovered as the trace of its tensorial generalization.

Considering the asymptotic limit of infinite times, the identity (2-11) further gives the generalized (tensorial) virial theorem, as the tensorial extension of the scalar virial theorem

$$
2\left\langle\hat{\boldsymbol{E}}_{k}\right\rangle+\langle\hat{\boldsymbol{V}}\rangle=\mathbf{0} .
$$

The virial can be decomposed into the sum of the internal virial $\hat{V}_{\text {int }}$ and the external virial $\hat{V}_{\text {ext }}$, as

$$
\hat{V}=\left(\boldsymbol{f}_{i j} \cdot \boldsymbol{r}_{i j}\right)_{j \neq i}+\boldsymbol{f}_{i, \mathrm{ext}} \cdot \boldsymbol{r}_{i} \equiv \hat{V}_{\mathrm{int}}+\hat{V}_{\mathrm{ext}}
$$

highlighting the contribution of internal forces $f_{i j}$ (first term on the right due to interparticle interactions) and external forces (body forces due to gravity and contact), denoted $\boldsymbol{f}_{i, \text { ext }}$, adopting the notation $\boldsymbol{r}_{i j}:=\boldsymbol{r}_{i}-\boldsymbol{r}_{j}$ for the relative position of particles $i$ and $j$.

\section{Scalar and tensorial viral theorems for systems with varying mass}

Variable mass problems have been treated in the literature in the context of the virial theorem [Gommerstadt 2001], especially considering applications in astronomy. The authors especially mention that when a body is losing mass isotropically, no additional force should appear, thus the motion of the body will overall not be altered by mass losses. 
The mass balance writes in integral form as

$$
\frac{D}{D t} \int_{\Omega} \rho d x=\int_{\Omega} \rho(\pi-\operatorname{div} \boldsymbol{J}) d x
$$

in presence of a source term $\pi$ and a mass flux vector $\boldsymbol{J}$, which can be identified for an open system including different chemical species as

$$
\pi=\sum_{k} \rho_{k} \dot{n}_{k}, \quad \boldsymbol{J}=\sum_{k} \boldsymbol{J}_{k} .
$$

In continuum mechanics, the balance of linear momentum is written in integral form as

$$
\begin{aligned}
\frac{D}{D t} \int_{\Omega} \rho \boldsymbol{v} d x & =\int_{\Omega} \boldsymbol{f} d x+\int_{\partial \Omega} \boldsymbol{n} \cdot \boldsymbol{\sigma} d s+\int_{\Omega} \pi \boldsymbol{v} d x-\int_{\partial \Omega} \boldsymbol{n} \cdot(\boldsymbol{J} \otimes \boldsymbol{v}) d s \\
& =\int_{\Omega} \boldsymbol{f} d x+\int_{\partial \Omega} \boldsymbol{n} \cdot \boldsymbol{\sigma} d s+\int_{\Omega} \pi \boldsymbol{v} d x-\int_{\Omega} \operatorname{div}(\boldsymbol{v} \otimes \boldsymbol{J}) d x \\
& \equiv \int_{\Omega} \boldsymbol{f} d x+\int_{\Omega} \pi \boldsymbol{v} d x+\int_{\Omega} \operatorname{div}(\boldsymbol{\sigma}-\boldsymbol{v} \otimes \boldsymbol{J}) d x .
\end{aligned}
$$

The last equality highlights that the effective stress is in fact the second order tensor

$$
\tilde{\sigma}:=\sigma-v \otimes J .
$$

Note that we have used the fact the divergence is presently elaborated as the right divergence operator. For a continuum body, the strong form of the mass and momentum balance laws in presence of mass changes are successively obtained as

and

$$
\frac{d \rho}{d t} \equiv \dot{\rho}=\pi-\nabla \cdot \boldsymbol{J}-\rho \nabla \boldsymbol{v}
$$

$$
\rho \frac{d \boldsymbol{v}}{d t}=\boldsymbol{f}+\nabla \cdot \boldsymbol{\sigma}-(\boldsymbol{J} \cdot \nabla) \boldsymbol{v} \equiv \boldsymbol{f}+\nabla \cdot \boldsymbol{\sigma}-\nabla \boldsymbol{v} \cdot \boldsymbol{J},
$$

that is to say

$$
\begin{aligned}
\rho \frac{d v_{i}}{d t} & =f_{i}+\sigma_{i p, p}-\frac{\partial v_{i}}{\partial x_{p}} J_{p} \\
& =f_{i}+\sigma_{i p, p}-J_{p} v_{i, p}=f_{i}+\left(\sigma_{i p}-v_{i} J_{p}\right)_{, p}+v_{i} \operatorname{div} \boldsymbol{J} .
\end{aligned}
$$

This entails the balance of linear momentum equality

$$
\rho \frac{d \boldsymbol{v}}{d t}=(\boldsymbol{f}+\boldsymbol{v} \operatorname{div} \boldsymbol{J})+\nabla \cdot \tilde{\boldsymbol{\sigma}} .
$$

We can see that the additional contribution $\boldsymbol{v} \operatorname{div} \boldsymbol{J}$ acts in fact as a source term in the balance of linear momentum (3-5), and can be incorporated into the overall effective body force, quantity $(\boldsymbol{f}+\boldsymbol{v} \operatorname{div} \boldsymbol{J})$. 
3.1. Cauchy stress from the discrete form of the virial theorem. The discrete scalar virial in eulerian form is built as the dyadic product of the spatial positions of the material points with the forces acting on them [Jouanna and Brocas 2001]:

$$
\hat{V}=\sum_{i=1}^{N} \boldsymbol{r}_{i} \cdot \boldsymbol{f}_{i}=\sum_{i, j=1}^{N}\left(\boldsymbol{f}_{i j} \cdot \boldsymbol{r}_{i j}\right)_{j \neq i}+\sum_{i=1}^{N_{\mathrm{ext}}} \boldsymbol{f}_{i, \mathrm{ext}} \cdot \boldsymbol{r}_{i} \equiv \hat{V}_{\mathrm{int}}+\hat{V}_{\mathrm{ext}},
$$

yielding

$$
\begin{aligned}
\langle\hat{V}\rangle=\left\langle\hat{V}_{\mathrm{int}}\right\rangle+\left\langle\hat{V}_{\mathrm{ext}}\right\rangle & \cong\left\langle\sum_{i=1}^{N_{\mathrm{con}}} r_{i} \cdot \boldsymbol{f}_{i, \mathrm{con}}\right\rangle+\left\langle\sum_{i=1}^{N_{\mathrm{ext}}} r_{i} \cdot \boldsymbol{f}_{i, \mathrm{vol}}\right\rangle \\
& =\int_{\partial \boldsymbol{\Omega}} \boldsymbol{r} \cdot \boldsymbol{\sigma} \cdot \boldsymbol{n} d \sigma_{t}+\int_{\boldsymbol{\Omega}} \boldsymbol{r} \cdot \boldsymbol{f} d x \\
& =\int_{\boldsymbol{\Omega}}\left(\boldsymbol{r} \cdot \operatorname{div} \boldsymbol{\sigma}+\boldsymbol{\sigma}^{t}: \operatorname{grad} \boldsymbol{r}\right) d x+\int_{\boldsymbol{\Omega}} \boldsymbol{r} \cdot \boldsymbol{f} d \sigma_{t} \\
& =\int_{\boldsymbol{\Omega}}\left(\boldsymbol{r} \cdot \operatorname{div} \boldsymbol{\sigma}+\boldsymbol{\sigma}^{t}: \boldsymbol{I}\right) d x+\int_{\boldsymbol{\Omega}} \boldsymbol{r} \cdot \boldsymbol{f} d x
\end{aligned}
$$

which we view as the ensemble average of the discrete (scalar) eulerian virial, denoted by $\langle V\rangle$. Since contact and external (body) forces do not act on the same material points, we have indicated in (3-6) the range of these respective material points by $N_{\text {cont }}$ and $N_{\text {ext }}$, respectively; this notation will be retained throughout.

The contribution $\int_{\partial \boldsymbol{\Omega}} \boldsymbol{r} \cdot \boldsymbol{\sigma} \cdot \boldsymbol{n} d \sigma_{t}$ in previous equality represents the exterior virial due to contact forces, considering that interactions between particles have a very short range, hence the particles contributing to the exernal virial are those located near the boundaries of the considered volume element. These forces are in fact contact forces (reflected in the existence of Cauchy stress at the continuum level), and thus are considered as internal forces corresponding to the internal virial $\hat{V}_{\text {int }}$. The contribution $\int_{\boldsymbol{\Omega}} \boldsymbol{r} \cdot \boldsymbol{f} d \sigma_{t}$ represents the contribution to the scalar virial due to external forces, and is accordingly coined the external virial, denoted $\hat{V}_{\text {ext }}$ in (3-6).

In the sequence of equalities in (3-6), we have used the analogy between the discrete and continuous counterpart of the scalar virial of external and contact (internal) forces. The second row of equalities in (3-6) is the continuous counterpart of the discrete elaboration of the scalar virial in (2-6), as previously explained: internal forces in a continuum mechanical description are identified to contact forces, while external forces are typically body forces or any force at distance.

Introducing therein the previous balance of momentum, rewritten here for the sake of clarity as

$$
\rho \frac{d \boldsymbol{v}}{d t}=\boldsymbol{f}+\nabla \cdot \sigma-(\boldsymbol{J} \cdot \nabla) \boldsymbol{v}
$$


leads further to

$$
\left\langle\sum_{i=1}^{N_{\mathrm{cont}}} r_{i} \cdot \boldsymbol{f}_{i, \mathrm{con}}\right\rangle+\left\langle\sum_{i=1}^{N_{\mathrm{ext}}} r_{i} \cdot \boldsymbol{f}_{i, \mathrm{vol}}\right\rangle=\int_{V} \boldsymbol{r} \cdot(\rho \boldsymbol{\gamma}+(\boldsymbol{J} . \nabla) \boldsymbol{v}) d V+\int_{V} \boldsymbol{\sigma}^{T}: \boldsymbol{I} d V .
$$

Introducing the acceleration $\boldsymbol{\gamma}:=d \boldsymbol{v} / d t$ therein. One can thus express the trace of Cauchy stress as

$$
\begin{aligned}
|V| I_{1}(\boldsymbol{\sigma}): & =|V| \operatorname{Tr}(\boldsymbol{\sigma}) \\
& =\left\langle\sum_{i=1}^{N_{\mathrm{cont}}} r_{i} \cdot \boldsymbol{f}_{i, \mathrm{con}}\right\rangle+\left\langle\sum_{i=1}^{N_{\mathrm{ext}}} r_{i} \cdot \boldsymbol{f}_{i, \mathrm{vol}}\right\rangle-\int_{V} \boldsymbol{r} \cdot(\rho \boldsymbol{\gamma}+(\boldsymbol{J} \cdot \nabla) \boldsymbol{v}) d V \\
& \cong\left\langle\sum_{i=1}^{N_{\mathrm{cont}}} r_{i} \cdot \boldsymbol{f}_{i, \mathrm{con}}\right\rangle+\left\langle\sum_{i=1}^{N_{\mathrm{ext}}} r_{i} \cdot \boldsymbol{f}_{i, \mathrm{vol}}\right\rangle-\int_{V} \boldsymbol{r} \cdot(\boldsymbol{J} \cdot \nabla) \boldsymbol{v} d V
\end{aligned}
$$

It is customary to neglect the inertia forces, so that the pressure now involves an additional contribution given by the last integral in previous equality, involving the mass flux. The last equality is the extended scalar virial theorem in Eulerian format accounting for mass changes within a body of a set of particles.

The tensor form of the virial theorem is obtained as follows:

$$
\begin{aligned}
\boldsymbol{V}_{\mathrm{ext}, \mathrm{tot}}=\boldsymbol{V}_{\mathrm{ext}, \mathrm{con}}+\boldsymbol{V}_{\mathrm{ext}, \mathrm{vol}} & \cong\left\langle\sum_{i=1}^{N_{\mathrm{cont}}} r_{i} \cdot \boldsymbol{f}_{i, \mathrm{con}}\right\rangle+\left\langle\sum_{i=1}^{N_{\mathrm{ext}}} r_{i} \cdot \boldsymbol{f}_{i, \mathrm{vol}}\right\rangle \\
& =\int_{\partial V} \boldsymbol{r} \otimes \boldsymbol{\sigma} \cdot \boldsymbol{n} d s+\int_{V} \boldsymbol{r} \otimes \boldsymbol{f}_{\mathrm{vol}} d x \\
& =\int_{V}\left(\boldsymbol{r} \otimes \operatorname{div} \boldsymbol{\sigma}+\boldsymbol{I} \cdot \boldsymbol{\sigma}^{T}\right) d x+\int_{V} \boldsymbol{r} \otimes \boldsymbol{f}_{\mathrm{vol}} d x
\end{aligned}
$$

Inserting the previous balance of linear momentum delivers the equality

$$
\begin{aligned}
\left\langle\sum_{i=1}^{N_{\mathrm{cont}}} r_{i} \cdot \boldsymbol{f}_{i, \mathrm{con}}\right\rangle & +\left\langle\sum_{i=1}^{N_{\mathrm{ext}}} r_{i} \cdot \boldsymbol{f}_{i, \mathrm{vol}}\right\rangle \\
& =\int_{V}\left(\boldsymbol{r} \otimes(\rho \boldsymbol{\gamma}-\boldsymbol{f}+(\boldsymbol{J} \cdot \nabla) \boldsymbol{v})+\boldsymbol{\sigma}^{T}\right) d x+\int_{V} \boldsymbol{r} \otimes \boldsymbol{f}_{\mathrm{vol}} d x .
\end{aligned}
$$

Neglecting body forces on both sides and inertia forces we can then obtain the average of the Cauchy stress tensor:

$$
\int_{V} \boldsymbol{\sigma}^{T} d x \approx|V| \boldsymbol{\sigma}^{T}=\left\langle\sum_{i=1}^{N} \boldsymbol{r}_{i} \otimes \boldsymbol{f}_{i, \mathrm{con}}\right\rangle-\int_{V}(\boldsymbol{r} \otimes(\rho \boldsymbol{\gamma}-\boldsymbol{f}+(\boldsymbol{J} \cdot \nabla) \boldsymbol{v})) d x,
$$


leading to

$$
\boldsymbol{\sigma}^{T}=\frac{1}{|V|}\left\langle\sum_{i=1}^{N_{\text {cont }}} \boldsymbol{r}_{i} \otimes \boldsymbol{f}_{i, \mathrm{con}}\right\rangle-\frac{1}{|V|} \int_{V} \boldsymbol{r} \otimes(\boldsymbol{J} \cdot \nabla) \boldsymbol{v} d x .
$$

We have considered a small enough volume so that the stress tensor can be considered as homogeneous inside. Thereby, Cauchy stress tensor is expressed versus the average virial of contact forces and the additional contribution of mass flux, in identified as the last integral in previous equality.

The average Cauchy stress can alternatively be derived from the continuum version of the virial theorem, as exposed in the next subsection. We shall in addition and as a matter of completeness incorporate the inertia forces, which have been neglected in previous derivations.

3.2. Continuum form of the virial theorem and average Cauchy stress. The full derivation of the virial theorem in scalar format and from a purely continuum viewpoint (that is without resorting to the discrete mechanics of a set of interacting particles) delivers the average of Cauchy stress as an extension of the virial theorem with constant mass (see for example identity (3) in [Gommerstadt 2001]) as

$$
\frac{1}{|V|} \int_{V} \boldsymbol{\sigma} d x=\frac{1}{|V|} \boldsymbol{E}_{c}-\frac{1}{2|V|} \frac{d^{2} \boldsymbol{I}}{d t^{2}}+\frac{1}{2|V|} \int_{\partial V} \boldsymbol{x} \otimes \boldsymbol{\sigma} \cdot \boldsymbol{n} d s .
$$

The tensor of kinetic energy therein is defined as

$$
\boldsymbol{E}_{c}:=\frac{1}{2} \int_{V} \rho \boldsymbol{v} \otimes \boldsymbol{v} d x
$$

The inertia tensor and its second material derivative are computed successively as follows:

$$
\begin{aligned}
\boldsymbol{I} & =\int_{V} \rho \boldsymbol{x} \otimes \boldsymbol{x} d x \\
\frac{d \boldsymbol{I}}{d t} & =\int_{V} \rho(\boldsymbol{x} \otimes \boldsymbol{v}+\boldsymbol{v} \otimes \boldsymbol{x}) d x+\int_{V} \frac{d \rho}{d t} \boldsymbol{x} \otimes \boldsymbol{x} d x+\int_{V} \rho(\boldsymbol{x} \otimes \boldsymbol{x}) \nabla \cdot \boldsymbol{v} d x \\
\frac{d^{2} \boldsymbol{I}}{d t} & =\int_{V} \rho(\boldsymbol{\gamma} \otimes \boldsymbol{x}+\boldsymbol{x} \otimes \boldsymbol{\gamma}+2 \boldsymbol{v} \otimes \boldsymbol{v}) d x+2 \int_{V}(\dot{\rho}+\rho \nabla \cdot \boldsymbol{v}) \frac{D}{D t}(\boldsymbol{x} \otimes \boldsymbol{x}) d x \\
& +\int_{V}\left(\ddot{\rho}+2 \dot{\rho} \nabla \cdot \boldsymbol{v}+\rho \frac{D}{D t}(\nabla \cdot \boldsymbol{v})+\rho(\nabla \cdot \boldsymbol{v})^{2}\right)(\boldsymbol{x} \otimes \boldsymbol{x}) d x .
\end{aligned}
$$

In the particular case of incompressible media, the condition $\nabla \cdot v=0$ entails the simplified expression of the second-order material derivative of the inertia tensor $\frac{d^{2} \boldsymbol{I}}{d t}=\int_{V} \rho(\boldsymbol{\gamma} \otimes \boldsymbol{x}+\boldsymbol{x} \otimes \boldsymbol{\gamma}+2 \boldsymbol{v} \otimes \boldsymbol{v}) d x+2 \int_{V} \dot{\rho} \frac{D}{D t}(\boldsymbol{x} \otimes \boldsymbol{x}) d x+\int_{V} \ddot{\rho}(\boldsymbol{x} \otimes \boldsymbol{x}) d x$ 
in which the second-order time derivative of the mass density results from balance law (3-4) incorporating mass source and mass flux contributions.

Inserting expression (3-14) into (3-12) then delivers the following expression for the average Cauchy stress tensor:

$$
\begin{aligned}
\overline{\boldsymbol{\sigma}}:= & \frac{1}{|V|} \int_{V} \boldsymbol{\sigma} d x \\
= & \frac{1}{|V|} \boldsymbol{E}_{c}+\frac{1}{2|V|} \int_{\partial V} \boldsymbol{x} \otimes \boldsymbol{\sigma} \cdot \boldsymbol{n} d s \\
& \quad-\frac{1}{2|V|}\left\{\int_{V} \rho(\boldsymbol{\gamma} \otimes \boldsymbol{x}+\boldsymbol{x} \otimes \boldsymbol{\gamma}+2 \boldsymbol{v} \otimes \boldsymbol{v}) d x+2 \int_{V}(\dot{\rho}+\rho \nabla \cdot \boldsymbol{v}) \frac{D}{D t}(\boldsymbol{x} \otimes \boldsymbol{x}) d x\right. \\
& \left.\quad+\int_{V}\left(\ddot{\rho}+2 \dot{\rho} \nabla \cdot \boldsymbol{v}+\rho \frac{D}{D t}(\nabla \cdot \boldsymbol{v})+\rho(\nabla \cdot \boldsymbol{v})^{2}\right)(\boldsymbol{x} \otimes \boldsymbol{x}) d x\right\} .
\end{aligned}
$$

Based on (3-15), this expression simplifies for incompressible media to deliver the full Cauchy stress tensor in averaged form:

$$
\begin{aligned}
\overline{\boldsymbol{\sigma}}:= & \frac{1}{|V|} \int_{V} \boldsymbol{\sigma} d x \\
= & \frac{1}{|V|} \boldsymbol{E}_{c}+\frac{1}{2|V|} \int_{\partial V} \boldsymbol{x} \otimes \boldsymbol{\sigma} \cdot \boldsymbol{n} d s \\
& \quad-\frac{1}{2|V|}\left\{\int_{V} \rho(\boldsymbol{\gamma} \otimes \boldsymbol{x}+\boldsymbol{x} \otimes \boldsymbol{\gamma}+2 \boldsymbol{v} \otimes \boldsymbol{v}) d x\right. \\
& \left.+2 \int_{V} \dot{\rho} \frac{D}{D t}(\boldsymbol{x} \otimes \boldsymbol{x}) d x+\int_{V} \ddot{\rho}(\boldsymbol{x} \otimes \boldsymbol{x}) d x\right\} .
\end{aligned}
$$

\section{Material version of the scalar virial theorem for systems with varying mass}

Since Cauchy stress represents a spatial measure of the contact forces in condensed matter, one expects a similar interpretation of the Eshelby stress, from the tensorial virial and extensions thereof, viewed as the material counterpart of Cauchy stress. Microscopic interpretations of the notion of Eshelby stress are of high interest, since this tensor leads to the so called material forces accounting for the presence of defects (inhomogeneities, such as inclusions or cracks) in material space [Maugin 1993]. Hence, discrete simulations in the configuration of the defects based on the virial can be conceived as a mean to evaluate those material forces at the very scale of the defect themselves.

Pursuing further along this line of thoughts, the construction of Eshelby stress from considerations tied to a system of discrete interacting punctual masses proves 
also relevant in the context of the so-called continuum-atomistic modeling strategies in multiscale simulation methods, see [Alibert et al. 2003; Sunyk and Steinmann 2003; Tadmor et al. 1996] and references therein. Such an interpretation of the stress tensor has been done in the continuum modeling of granular materials such as sands, cements, clays, concrete, rocks and certain polymers [Misra and Singh 2015; Misra and Poorsolhjouy 2015b; 2015a].

We establish the material version of the scalar virial theorem; we adopt as for the eulerian situation a quasi-static framework, and define the scalar material virial $\hat{V}_{0}$ with ensemble average $\left\langle\hat{V}_{0}\right\rangle$ as [Ganghoffer 2010b]

$$
\begin{aligned}
\left\langle\hat{V}_{0}\right\rangle & :=-\int_{\partial \boldsymbol{\Omega}_{0}} \boldsymbol{R} \cdot \boldsymbol{\Sigma} \cdot \boldsymbol{N} d \sigma_{0}+\int_{\boldsymbol{\Omega}_{0}} \boldsymbol{R} \cdot \boldsymbol{f}_{R} d X \\
& \equiv-\int_{\boldsymbol{\Omega}_{0}} \boldsymbol{I}: \boldsymbol{\Sigma}^{t} d X \cong-\operatorname{Tr}(\boldsymbol{\Sigma})\left|\boldsymbol{\Omega}_{0}\right|
\end{aligned}
$$

considering a small enough volume element $\boldsymbol{\Omega}_{0}$, so that the fields can be considered as nearly homogeneous (equilibrium in terms of Eshelby stress has been used); vector $\boldsymbol{R}$ is the material position. Previous identity has been obtained by a pull-back of the eulerian form of the balance of linear momentum on the material manifold.

An elaboration of the scalar material virial can be done alternatively starting from a construction similar to that of the eulerian scalar virial in (2-6) for its discrete version or in (3-6) for the continuum counterpart: we define the scalar material virial as the dot product of the spatial positions of material points with the forces acting on them (with a change of sign for the internal virial of contact forces); developments presented in [Ganghoffer 2010b] lead to

$$
\hat{V}_{R}=\sum_{i=1}^{N} \boldsymbol{R}_{i} \cdot \boldsymbol{f}_{R i},
$$

which is equivalent to

$$
\begin{aligned}
\left\langle\hat{V}_{R}\right\rangle & :=-\int_{\partial \boldsymbol{\Omega}_{R}} \boldsymbol{R} \cdot \boldsymbol{\Sigma} \cdot \boldsymbol{N} d \sigma_{R}+\int_{\boldsymbol{\Omega}} \boldsymbol{R} \cdot \boldsymbol{f}_{R} d X \\
& =-\int_{\boldsymbol{\Omega}}\left(\boldsymbol{R} \cdot \nabla_{R} \cdot \boldsymbol{\Sigma}+\boldsymbol{\Sigma}^{t}: \nabla_{R} \boldsymbol{R}\right) d x+\int_{\boldsymbol{\Omega}} \boldsymbol{R} \cdot \boldsymbol{f}_{R} d \sigma_{t} \\
& =-\int_{\boldsymbol{\Omega}}\left(\boldsymbol{R} \cdot \nabla_{R} \cdot \boldsymbol{\Sigma}+\boldsymbol{\Sigma}^{t}: \boldsymbol{I}\right) d x+\int_{\boldsymbol{\Omega}} \boldsymbol{R} \cdot \boldsymbol{f}_{R} d x .
\end{aligned}
$$

In previous set of equalities, the index $R$ refers to the referential configuration; thus one has the identity $\hat{V}_{R}=\hat{V}_{0}$.

Inserting the material divergence of the Eshelby tensor $\Sigma$, previously obtained as

$$
\nabla_{R} \cdot \boldsymbol{\Sigma}=\nabla_{R} \cdot \tilde{\boldsymbol{\Sigma}}+\boldsymbol{J}_{R} \cdot \nabla_{R} \boldsymbol{F} \cdot \boldsymbol{v}+\nabla_{R} \cdot\left(\boldsymbol{F}^{T} \cdot\left(\boldsymbol{J}_{R} \otimes \boldsymbol{v}\right)\right)
$$


delivers the trace of Eshelby stress as

$$
\begin{aligned}
-|\boldsymbol{\Omega}| \operatorname{Tr}\left(\boldsymbol{\Sigma}^{T}\right) & =\int_{\boldsymbol{\Omega}}\left(\boldsymbol{R} \cdot \nabla_{R} \cdot \boldsymbol{\Sigma}\right) d x+\int_{\boldsymbol{\Omega}} \boldsymbol{R} \cdot \boldsymbol{f}_{R} d x-\left\langle\hat{V}_{R}\right\rangle \\
& =\int_{\boldsymbol{\Omega}}\left(\boldsymbol{R} \cdot \nabla_{R} \cdot \tilde{\boldsymbol{\Sigma}}\right) d x+\int_{\boldsymbol{\Omega}} \boldsymbol{R} \cdot\left\{\boldsymbol{J}_{R} \cdot \nabla_{R} \boldsymbol{F} \cdot \boldsymbol{v}+\nabla_{R} \cdot\left(\boldsymbol{F}^{T} \cdot\left(\boldsymbol{J}_{R} \otimes \boldsymbol{v}\right)\right)\right\} d x \\
& +\int_{\boldsymbol{\Omega}} \boldsymbol{R} \cdot \boldsymbol{f}_{R} d x-\left\langle\hat{V}_{R}\right\rangle .
\end{aligned}
$$

Inserting therein the balance of momentum with the effective Eshelby stress finally delivers the trace of Eshelby stress versus the source of mass terms, the referential scalar virial, and the heat and chemical contributions:

$$
\begin{array}{r}
-|\Omega| \operatorname{Tr}\left(\boldsymbol{\Sigma}^{t}\right)=-\int_{\Omega}\left(\boldsymbol{R} \cdot\left\{\Pi \boldsymbol{F}^{T} \cdot \boldsymbol{v}+\nabla_{R} \cdot\left[\boldsymbol{F}^{T} \cdot \tilde{\boldsymbol{T}}\right]+s \nabla_{R} \theta+\mu_{k} \nabla_{R} n_{k}\right\}\right) d x \\
+\int_{\Omega} \boldsymbol{R} \cdot\left\{\nabla_{R} \cdot\left(\boldsymbol{F}^{T} \cdot\left(\boldsymbol{J}_{R} \otimes \boldsymbol{v}\right)\right)\right\} d x-\left\langle\hat{V}_{R}\right\rangle .
\end{array}
$$

Microscopic interpretations of the notion of Eshelby stress are of high interest, since this tensor leads to the so-called material forces accounting for the presence of defects (inhomogeneities, such as inclusions or cracks) in material space [Maugin 1993]. Hence, discrete simulations in the configuration of the defects based on the virial can be conceived as a mean to evaluate those material forces at the very scale of the defect themselves. Since the virial relies on the consideration of a discrete set of interacting particles, one may further evoke the mixed continuumatomistic approaches that prove adequate in nanomechanics, which combine the usual framework of continuum mechanics with a full atomic scale description based on interatomic potentials. The full Eshelby stress shall be derived in the next section from the virial theorem.

\section{Eshelby stress for continua with variable mass}

Recall that the tensorial virial states [Jouanna and Brocas 2001] that the transpose of the Cauchy stress may be expressed as the average external virial tensor divided by the volume occupied by the set of considered particles. The external tensorial virial is defined as the contribution of the tensorial virial due exclusively to the external forces $\boldsymbol{f}_{i, \mathrm{ext}}$ :

$$
\hat{V}_{\mathrm{ext}}:=\boldsymbol{r}_{i} \otimes \boldsymbol{f}_{i, \mathrm{ext}}
$$

It is the principal aim of this section to give a similar microscopic interpretation of the purely material Eshelby stress in terms of the material counterpart of the tensorial virial, to be elaborated in the sequel. 
Starting from the tensorial eulerian virial as the following integral [Jouanna and Brocas 2001], adopting the continuum limit, viz

$$
\langle\hat{\boldsymbol{V}}\rangle \equiv \int_{\partial \boldsymbol{\Omega}} \boldsymbol{r} \otimes \boldsymbol{\sigma} \cdot \boldsymbol{n} d \sigma_{t}+\int_{\boldsymbol{\Omega}} \boldsymbol{r} \otimes \boldsymbol{f} d x .
$$

And accounting for the relation between the spatial and material tensorial virials

$$
\langle\hat{\boldsymbol{V}}\rangle=\left\langle\hat{\boldsymbol{V}}_{0}\right\rangle+\int_{\boldsymbol{\Omega}_{0}} W_{0} \boldsymbol{I} d X
$$

one obtains after length developments presented in the Appendix B the average of the Eshelby stress versus the eulerian tensorial virial:

$$
\langle\hat{\boldsymbol{V}}\rangle=\int_{\boldsymbol{\Omega}_{0}}\left(-\operatorname{tr}\left(\tilde{\tilde{\boldsymbol{\Sigma}}}^{t}\right)+W_{0} \boldsymbol{I}\right) d X+\int_{\boldsymbol{\Omega}_{0}} \boldsymbol{R} \cdot\left\{\boldsymbol{F}^{T} \otimes \rho J \frac{d \boldsymbol{v}}{d t}+\boldsymbol{F}^{T} \otimes J(\boldsymbol{J} \cdot \nabla) \boldsymbol{v}\right\} d X,
$$

leading to

$$
\begin{aligned}
\int_{\boldsymbol{\Omega}_{0}} \operatorname{tr}\left(\tilde{\tilde{\Sigma}}^{t}\right) d X & \equiv \int_{\boldsymbol{\Omega}_{0}} \boldsymbol{\Sigma} d X \\
& =-\left\langle\hat{\boldsymbol{V}}_{0}\right\rangle+\int_{\boldsymbol{\Omega}_{0}} \boldsymbol{R} \cdot\left\{\boldsymbol{F}^{T} \otimes \rho J \frac{d \boldsymbol{v}}{d t}+\boldsymbol{F}^{T} \otimes J(\boldsymbol{J} \cdot \nabla) \boldsymbol{v}\right\} d X .
\end{aligned}
$$

The averaged material virial therein satisfies the material version of the tensorial virial theorem (for asymptotic times), which is the equality

$$
\left\langle\hat{\boldsymbol{V}}_{0}\right\rangle=\left\langle\hat{\boldsymbol{V}}_{0 \text { ext,tot }}\right\rangle+\left\langle\hat{\boldsymbol{V}}_{0 \text { int }}\right\rangle, \quad\left\langle\hat{\boldsymbol{V}}_{0 \text { ext, tot }}\right\rangle+\left\langle\hat{\boldsymbol{V}}_{0 \text { int }}\right\rangle+2\left\langle\hat{\boldsymbol{E}}_{k}\right\rangle=\mathbf{0} .
$$

This writing leads to the following expression of the average Eshelby stress

$$
\begin{aligned}
\overline{\boldsymbol{\Sigma}} & :=\frac{1}{\left|\boldsymbol{\Omega}_{0}\right|} \int_{\boldsymbol{\Omega}_{0}} \boldsymbol{\Sigma} d X \\
& =-\frac{1}{\left|\boldsymbol{\Omega}_{0}\right|}\left\langle\hat{\boldsymbol{V}}_{0}\right\rangle+\frac{1}{\left|\boldsymbol{\Omega}_{0}\right|} \int_{\boldsymbol{\Omega}_{0}} \boldsymbol{R} \cdot\left\{\boldsymbol{F}^{T} \otimes \rho J \frac{d \boldsymbol{v}}{d t}+\boldsymbol{F}^{T} \otimes J(\boldsymbol{J} \cdot \nabla) \boldsymbol{v}\right\} d X \\
& =\frac{1}{\left|\boldsymbol{\Omega}_{0}\right|}\left\{\left\langle\hat{\boldsymbol{V}}_{0} \text { int }\right\rangle+2\left\langle\boldsymbol{E}_{k}\right\rangle\right\}+\frac{1}{\left|\boldsymbol{\Omega}_{0}\right|} \int_{\boldsymbol{\Omega}_{0}} \boldsymbol{R} \cdot\left\{\boldsymbol{F}^{T} \otimes \rho J \frac{d \boldsymbol{v}}{d t}+\boldsymbol{F}^{T} \otimes J(\boldsymbol{J} \cdot \nabla) \boldsymbol{v}\right\} d X
\end{aligned}
$$

the right-hand side being evaluated using the discrete expression of the internal virial and kinetic energy (averaged over long times); the internal virial results from the additive decomposition of the total tensor virial into the internal and external virials,

$$
\hat{\boldsymbol{V}}=\left(\boldsymbol{r}_{i j} \otimes \boldsymbol{f}_{i j}\right)_{j \neq i}+\boldsymbol{r}_{i} \otimes f_{i, \mathrm{ext}} \equiv \hat{\boldsymbol{V}}_{\mathrm{int}}+\hat{\boldsymbol{V}}_{\mathrm{ext}},
$$

which leads to

$$
\hat{\boldsymbol{V}}_{0 \text { int }} \equiv\left(\boldsymbol{r}_{i j} \otimes \boldsymbol{F}^{T} \cdot \boldsymbol{f}_{i j}\right)_{j \neq i}
$$


adopting the following definition for the total discrete tensorial virial:

$$
\hat{\boldsymbol{V}}_{0}=\sum_{i=1}^{N} \boldsymbol{R}_{i} \otimes \boldsymbol{F}^{t} \cdot \boldsymbol{f}_{0 i} .
$$

Expression (5-6) involves the pull-back to the material manifold of the referential internal (traducing interactions between particles within the considered domain) forces $\boldsymbol{F}^{T} \cdot \boldsymbol{f}_{i j}$, accounting for both contact and volumetric forces.

Observe that the introduced fourth-order Eshelby tensor $\tilde{\tilde{\Sigma}}$ involved in previous derivations is an intermediate object originating from the mathematical developments initiated from the tensorial eulerian virial, which finally reduces to the classical (second order) Eshelby tensor by taking the trace of $\tilde{\tilde{\Sigma}}$.

The balance of momentum satisfied by the effective Eshelby stress

$$
\tilde{\boldsymbol{\Sigma}}:=W \boldsymbol{I}-\boldsymbol{F}^{T} \cdot \tilde{\boldsymbol{T}}
$$

, built from $\tilde{\boldsymbol{T}}$, is derived in Appendix A, leading to equality (A-12), which is recalled for completeness:

$$
\rho_{R} \boldsymbol{F}^{T} \cdot \frac{\partial \boldsymbol{v}}{\partial t}=\boldsymbol{f}_{R}+\Pi \boldsymbol{F}^{T} \cdot \boldsymbol{v}+\nabla_{R} \boldsymbol{F}:\left(\boldsymbol{J}_{R} \otimes \boldsymbol{v}\right)-\nabla_{R} \cdot \tilde{\boldsymbol{\Sigma}}+\left(\partial_{X} \psi\right)_{\exp l} .
$$

In (5-8), the quantity $\Pi \boldsymbol{F}^{T} \cdot \boldsymbol{v}+\boldsymbol{J}_{R} \cdot \nabla_{R} \boldsymbol{F} \cdot \boldsymbol{v}+\nabla_{R} \boldsymbol{F}:\left(\boldsymbol{J}_{R} \otimes \boldsymbol{v}\right)$ reflecting mass production and mass flux would vanish for closed systems with constant mass, in addition to modified Eshelby stress $\tilde{\boldsymbol{\Sigma}}$ coinciding with the classical Eshelby stress $\boldsymbol{\Sigma}$.

\section{Conclusion}

We have derived formal expressions of the Cauchy and Eshelby stress tensors for continuum bodies with varying mass, a situation of interest for growing solid bodies or for gravitational masses subjected to accretion phenomena. The adopted methodology relies on an extension of the virial theorem to situations of non constant mass, traduced by a mass flux through the system boundaries and a mass production term. These two additional contributions entail modifications of the balance of momentum, when considering either a spatial formulation involving Cauchy stress or a material formulation relying on Eshelby stress. The stress measures in both material and physical format have been expressed versus the tensorial virial, highlighting an additional contribution from the mass flux.

The present study shed some new light on the microscopic interpretation of Cauchy and Eshelby stress for systems with variable mass, bridging the gap between the microscopic (particle level) and the macroscopic continuum scales. Interpretation of those results from the microscopic or molecular point of view highlights 
that stresses may identified as the average of the virial tensor with additional contributions arising from the mass flux of particles entering the system, their evaluation resulting from the solution of the (dynamical in general) equations of motion at the microscopic or atomic level. This strategy may prove a convenient way to evaluate Eshelby and Cauchy stresses from discrete quantities, such as in finite element calculations (numerical approximation of a continuum model) or in simulations involving a two scale approach, like mixed atomistic continuum formulations. This approach appears of great interest in nanoscale systems with varying number of atoms due, for instance, to epitaxial growth, based on the extensive use of molecular dynamical simulations to explore the behavior of systems of atoms and molecules.

\section{Appendix A. Balance of momentum satisfied by the effective Eshelby stress}

The material form of the mass balance equation writes [Epstein and Maugin 2000],

$$
\frac{\partial \rho_{R}}{\partial t}=\Pi-\operatorname{Div} \boldsymbol{J}_{R}
$$

with the Lagrangian source and mass fluxes, respectively quantities $\Pi$ and $\boldsymbol{J}_{R}$, given versus their spatial counterparts as

$$
\Pi=J \pi, \quad J=J^{-1} \boldsymbol{F} \cdot J_{R}
$$

with the Jacobian $J:=\operatorname{det}(\boldsymbol{F})$. The Lagrangian balance of momentum expresses in terms of the nominal stress $\boldsymbol{T}$, the first Piola-Kirchoff stress tensor, as

$$
\rho_{R} \frac{\partial \boldsymbol{v}}{\partial t}=\boldsymbol{f}+\nabla_{R} \cdot \boldsymbol{T}-\left(\boldsymbol{J}_{R} \cdot \nabla_{R}\right) \boldsymbol{v}
$$

which rewrites accounting for the mass balance equation (A-1) as the dynamical equilibrium

$$
\rho_{R} \frac{\partial \boldsymbol{v}}{\partial t}=\boldsymbol{f}+\Pi \boldsymbol{v}+\nabla_{R} \cdot\left(\boldsymbol{T}-\boldsymbol{J}_{R} \otimes \boldsymbol{v}\right) .
$$

The balance of angular momentum expresses as the symmetry condition for the second-order tensor $\tilde{\boldsymbol{T}}:=\boldsymbol{T}-\boldsymbol{J}_{R} \otimes \boldsymbol{v}$, called the effective first Piola-Kirchhoff stress.

The material version of the balance of momentum is obtained by a pull-back of the eulerian version, using the relations [Milstein 1982]

$$
\operatorname{Div}\left(J \boldsymbol{F}^{-T}\right)=\mathbf{0} \quad \Rightarrow \quad \nabla_{R} \cdot \boldsymbol{T}=J \nabla \cdot \sigma,
$$

leading to

$$
\begin{aligned}
\rho_{R} \boldsymbol{F}^{T} \cdot \frac{\partial \boldsymbol{v}}{\partial t} & =\boldsymbol{F}^{T} \cdot \boldsymbol{f}+\Pi \boldsymbol{F}^{T} \cdot \boldsymbol{v}+\boldsymbol{F}^{T} \cdot \nabla_{R} \cdot \tilde{\boldsymbol{T}} \\
& =\boldsymbol{F}^{T} \cdot \boldsymbol{f}+\Pi \boldsymbol{F}^{T} \cdot \boldsymbol{v}+\boldsymbol{F}^{T} \cdot \nabla_{R} \cdot \boldsymbol{T}-\boldsymbol{F}^{T} \cdot \nabla_{R} \cdot\left(\boldsymbol{J}_{R} \otimes \boldsymbol{v}\right) .
\end{aligned}
$$


For a hyperelastic medium with strain energy density $W=W(\boldsymbol{F} ; X)$, consideration of the following identity for the total spatial material derivative of $W$ [Maugin 1993]

$$
d_{X^{A}} W=\frac{\partial W}{\partial F_{I}^{i}} F_{I, A}^{i}+\left(\frac{\partial W}{\partial X^{A}}\right)_{\exp l} \equiv T_{i}^{I} F_{I, A}^{i}+\left(\frac{\partial W}{\partial X^{A}}\right)_{\exp l}
$$

leads to

$$
\boldsymbol{F}^{T} \cdot \nabla_{R} \cdot \boldsymbol{T}=\nabla_{R} \cdot\left[\boldsymbol{F}^{T} \cdot \boldsymbol{T}-W \boldsymbol{I}\right]+\left(\boldsymbol{\partial}_{X} W\right)_{\exp l} .
$$

Due further to the equality

$$
\begin{aligned}
\boldsymbol{F}^{T} \cdot \nabla_{R} \cdot\left(\boldsymbol{J}_{R} \otimes \boldsymbol{v}\right) & =\boldsymbol{F}^{T} \cdot\left\{\boldsymbol{J}_{R} \cdot\left(\nabla_{R} \cdot \boldsymbol{v}\right)+\nabla_{R} \boldsymbol{J}_{R} \cdot \boldsymbol{v}\right\} \\
& =\left(\nabla_{R} \cdot \boldsymbol{v}\right) \boldsymbol{F}^{T} \cdot \boldsymbol{J}_{R}+\boldsymbol{F}^{T} \cdot \nabla_{R} \boldsymbol{J}_{R} \cdot \boldsymbol{v}
\end{aligned}
$$

one easily obtains

$$
\nabla_{R} \cdot\left[\boldsymbol{F}^{T} \cdot \tilde{\boldsymbol{T}}-W \boldsymbol{I}\right]=\nabla_{R} \cdot\left[\boldsymbol{F}^{T} \cdot \boldsymbol{T}-\boldsymbol{F}^{T} \cdot \boldsymbol{J}_{R} \otimes \boldsymbol{v}-W \boldsymbol{I}\right],
$$

with

$$
\begin{aligned}
\left(\boldsymbol{F}^{T} \cdot \tilde{T}\right)_{i j, j} & =\left(F_{k i} \tilde{T}_{k j}\right)_{, j}=F_{k i, j} \tilde{T}_{k j}+F_{k i} \tilde{T}_{k j, j} \\
& =\left(F_{k i, j} T_{k j}+F_{k i} T_{k j, j}\right)-J_{R k} F_{k i, j} v_{j}+F_{k i}\left(\nabla_{R} \cdot\left(\boldsymbol{J}_{R} \otimes \boldsymbol{v}\right)\right)_{k j},
\end{aligned}
$$

and hence the equality

$$
-\nabla_{R} \cdot \tilde{\Sigma}=-\nabla_{R} \cdot \Sigma-J_{R} \cdot \nabla_{R} \boldsymbol{F} \cdot \boldsymbol{v}-\nabla_{R} \cdot\left(\boldsymbol{F}^{T} \cdot\left(\boldsymbol{J}_{R} \otimes \boldsymbol{v}\right)\right)
$$

involving the Eshelby stress and modified Eshelby stress built from the hyperelastic potential $W$ :

$$
\boldsymbol{\Sigma}:=W \boldsymbol{I}-\boldsymbol{F}^{T} \cdot \boldsymbol{T}, \quad \tilde{\boldsymbol{\Sigma}}:=W \boldsymbol{I}-\boldsymbol{F}^{T} \cdot \tilde{\boldsymbol{T}} .
$$

More general similar derivations including chemical and thermal effects have been obtained in [Ganghoffer 2010b].

We further elaborate the dynamical equilibrium as

$$
\begin{aligned}
\rho_{R} \boldsymbol{F}^{T} \cdot \frac{\partial \boldsymbol{v}}{\partial t} & =\boldsymbol{F}^{T} \cdot \boldsymbol{f}+\Pi \boldsymbol{F}^{T} \cdot \boldsymbol{v}+\boldsymbol{F}^{T} \cdot \nabla_{R} \cdot \tilde{\boldsymbol{T}} \\
& \equiv \boldsymbol{F}^{T} \cdot \boldsymbol{f}+\Pi \boldsymbol{F}^{T} \cdot \boldsymbol{v}+\boldsymbol{F}^{T} \cdot \nabla_{R} \cdot \boldsymbol{T}-\boldsymbol{F}^{T} \cdot \nabla_{R} \cdot\left(\boldsymbol{J}_{R} \otimes \boldsymbol{v}\right), \\
\rho_{R} \boldsymbol{F}^{T} \cdot \frac{\partial \boldsymbol{v}}{\partial t} & =\boldsymbol{f}_{R}+\Pi \boldsymbol{F}^{T} \cdot \boldsymbol{v}-\nabla_{R} \cdot \boldsymbol{\Sigma}+\left(\partial_{X} \psi\right)_{\exp l}-\boldsymbol{F}^{T} \cdot \nabla_{R} \cdot\left(\boldsymbol{J}_{R} \otimes \boldsymbol{v}\right),
\end{aligned}
$$

which is equivalent to

$\rho_{R} \boldsymbol{F}^{T} \cdot \frac{\partial \boldsymbol{v}}{\partial t}$

$$
=\boldsymbol{f}_{R}+\Pi \boldsymbol{F}^{T} \cdot \boldsymbol{v}-\nabla_{R} \cdot \tilde{\boldsymbol{\Sigma}}+\left(\partial_{X} \psi\right)_{\exp l}+\nabla_{R} \cdot\left\{\boldsymbol{F}^{T} \cdot\left(\boldsymbol{J}_{R} \otimes \boldsymbol{v}\right)\right\}-\boldsymbol{F}^{T} \cdot \nabla_{R} \cdot\left(\boldsymbol{J}_{R} \otimes \boldsymbol{v}\right),
$$


or again to the equality

$$
\rho_{R} \boldsymbol{F}^{T} \cdot \frac{\partial \boldsymbol{v}}{\partial t}=\boldsymbol{f}_{R}+\Pi \boldsymbol{F}^{T} \cdot \boldsymbol{v}-\nabla_{R} \cdot \tilde{\boldsymbol{\Sigma}}+\nabla_{R} \boldsymbol{F}:\left(\boldsymbol{J}_{R} \otimes \boldsymbol{v}\right)+\left(\partial_{X} \psi\right)_{\exp l},
$$

involving the referential body forces $\boldsymbol{f}_{R}:=\boldsymbol{F}^{T} \cdot \boldsymbol{f}$. The modified effective Eshelby stress in (A-12) is the purely material stress incorporating the mass flux contribution.

\section{Appendix B. Derivation of the average Eshelby stress from the virial theorem}

The starting point is the relation (5-2), which is rewritten for the sake of clarity as

$$
\langle\hat{\boldsymbol{V}}\rangle \equiv \int_{\partial \boldsymbol{\Omega}} \boldsymbol{r} \otimes \boldsymbol{\sigma} \cdot \boldsymbol{n} d \sigma_{t}+\int_{\boldsymbol{\Omega}} \boldsymbol{r} \otimes \boldsymbol{f} d x .
$$

We then analyse the dyadic moment of physical forces - in the vocabulary of [Steinmann 2000] — therein:

$$
\begin{aligned}
\int_{\partial \boldsymbol{\Omega}} \boldsymbol{r} \otimes \boldsymbol{\sigma} \cdot \boldsymbol{n} d \sigma_{t} & \equiv \int_{\partial \boldsymbol{\Omega}} r_{i} \sigma_{j k} n_{k} d \sigma_{t}=\int_{\boldsymbol{\Omega}}\left(r_{i} \sigma_{j k}\right)^{\prime} k d x=\int_{\boldsymbol{\Omega}}\left(r_{i^{\prime} k} \sigma_{j k}+r_{i} \sigma_{j k, k}\right) d x \\
& \equiv \int_{\boldsymbol{\Omega}}\left(\operatorname{grad} \boldsymbol{r} \cdot \boldsymbol{\sigma}^{T}+\boldsymbol{r} \otimes \operatorname{div} \boldsymbol{\sigma}\right) d x \\
& =\int_{\boldsymbol{\Omega}} \boldsymbol{I} \cdot \boldsymbol{\sigma}^{T} d x+\int_{\boldsymbol{\Omega}} \boldsymbol{r} \otimes \operatorname{div} \boldsymbol{\sigma} d x
\end{aligned}
$$

Hence, assembling both contributions in $\langle\hat{\boldsymbol{V}}\rangle$ gives

$$
\langle\hat{\boldsymbol{V}}\rangle \equiv \int_{\boldsymbol{\Omega}} \boldsymbol{I} \cdot J \boldsymbol{\sigma}^{T} d X+\int_{\boldsymbol{\Omega}_{0}} \boldsymbol{R} \cdot\left\{\boldsymbol{F}^{T} \otimes J \operatorname{div} \boldsymbol{\sigma}+\boldsymbol{F}^{T} \otimes \boldsymbol{f}_{0}\right\} d X
$$

recalling that $\boldsymbol{f}_{0}:=J \boldsymbol{f}$.

A material form of static equilibrium shall next be expressed, obtained by transforming the integrand in (B-3) in a Lagrangian format. As a first step, the identity

$$
\boldsymbol{F}^{T} \otimes \operatorname{Div} \boldsymbol{T}=\operatorname{Div}\left(\boldsymbol{F}^{T} \otimes \boldsymbol{T}\right)-\operatorname{Grad}\left(W_{0} \boldsymbol{I}\right)
$$

is easily obtained, with

$$
\operatorname{Grad}\left(W_{0} \boldsymbol{I}\right)=\operatorname{Grad} \boldsymbol{F}^{T} \cdot \boldsymbol{T} \equiv\left(F^{t}\right)_{A i, B} T_{j B},
$$

the contraction being done on the material subscript $B$; observe that this relation is the tensorial generalization of the identity

$$
\operatorname{Div}\left(W_{0} \boldsymbol{I}\right)=\operatorname{Div} \boldsymbol{F}^{T} \cdot \boldsymbol{T} .
$$

Let us further express the gradient $\operatorname{Grad}(W I)$ above as the material divergence of 
a fourth-order tensor: due to the equality

$$
\boldsymbol{I}_{4}: \boldsymbol{A}=\operatorname{Tr}(\boldsymbol{A}) \boldsymbol{I}
$$

there follows the identity [Ganghoffer 2010b]

$$
\operatorname{Div}\left(W_{0} \boldsymbol{I}^{4}\right)=\operatorname{Grad}\left(W_{0} \boldsymbol{I}\right) .
$$

Let Div denote the material divergence, not to be confused with the spatial divergence operator, $\nabla$. From the classical Piola identity [Maugin 1993]

$$
\operatorname{div}\left(J \boldsymbol{F}^{-T}\right)=\mathbf{0}
$$

there follows further the relation )

$$
J \nabla \cdot \tilde{\boldsymbol{\sigma}}^{T}=\operatorname{Div} \tilde{\boldsymbol{T}}^{T}
$$

, with

$$
\tilde{\boldsymbol{T}}=J \tilde{\boldsymbol{\sigma}} \cdot \boldsymbol{F}^{-T} \equiv \boldsymbol{T}-J(\boldsymbol{J} \otimes \boldsymbol{v}) \cdot \boldsymbol{F}^{-T}
$$

the effective first Piola-Kirchhoff stress tensor. Combining the last identities with the Eulerian balance of linear momentum (3-5) yields

$$
\rho J \frac{d \boldsymbol{v}}{d t}=(J \boldsymbol{f}+\boldsymbol{v} J \operatorname{div} \boldsymbol{J})+J \nabla \cdot \tilde{\boldsymbol{\sigma}}^{T},
$$

which is to say

$$
\rho J \frac{d \boldsymbol{v}}{d t}=\tilde{f}_{0}+\operatorname{Div} \tilde{\boldsymbol{T}}^{T}
$$

with $\tilde{f}_{0}:=\left(\boldsymbol{f}_{0}+\boldsymbol{v} J \operatorname{div} \boldsymbol{J}\right)$ the effective body forces, and recalling the expression $\tilde{\boldsymbol{\sigma}}:=\boldsymbol{\sigma}-\boldsymbol{v} \otimes \boldsymbol{J}$ of the effective Cauchy stress.

The balance of linear momentum, (B-7), is further elaborated as

$$
\boldsymbol{F}^{T} \otimes \rho J \frac{d \boldsymbol{v}}{d t}=\boldsymbol{F}^{T} \otimes \tilde{\boldsymbol{f}}_{0}+\boldsymbol{F}^{T} \otimes \operatorname{Div} \tilde{\boldsymbol{T}}^{T} .
$$

Due further to the relation satisfied by the (classical) first Piola-Kirchhoff stress tensor,

$$
\boldsymbol{F}^{T} \otimes \operatorname{Div} \boldsymbol{T}=\operatorname{Div}\left(\boldsymbol{F}^{T} \otimes \boldsymbol{T}\right)-\operatorname{Grad}\left(W_{0} \boldsymbol{I}\right) \equiv \operatorname{Div}\left(\boldsymbol{F}^{T} \otimes \boldsymbol{T}-W_{0} \boldsymbol{I}^{4}\right),
$$

there follows the dynamical tensorial equilibrium equation

$$
\boldsymbol{F}^{T} \otimes \rho J \frac{d \boldsymbol{v}}{d t}=\boldsymbol{F}^{T} \otimes \tilde{f}_{0}-\operatorname{Div} \tilde{\tilde{\boldsymbol{\Sigma}}}-\boldsymbol{F}^{T} \otimes \operatorname{Div}\left\{J(\boldsymbol{J} \otimes \boldsymbol{v}) \cdot \boldsymbol{F}^{-T}\right\}
$$

involving the fourth-order material Eshelby tensor (denoted by a double tilde)

$$
\tilde{\tilde{\Sigma}}:=W_{0} I^{4}-\boldsymbol{F}^{T} \otimes \boldsymbol{T} .
$$


Adopting

$$
\operatorname{tr}(\boldsymbol{A} \otimes \boldsymbol{B}):=\boldsymbol{A} \cdot \boldsymbol{B}, \forall \boldsymbol{A}, \boldsymbol{B}
$$

as the definition of the trace of a fourth-order tensor built as the dyadic product of two second-order tensors, the trace of the fourth-order Eshelby tensor yields the second-order Eshelby tensor

$$
\Sigma:=W_{0} \boldsymbol{I}-\boldsymbol{F}^{T} \cdot \boldsymbol{T} .
$$

It is easy to show that $\boldsymbol{\Sigma}$ satisfies the following dynamical balance of linear momentum incorporating the mass flux:

$$
\rho J \boldsymbol{F}^{T} \cdot \frac{d \boldsymbol{v}}{d t}=\boldsymbol{F}^{T} \cdot \tilde{\boldsymbol{f}}_{0}-\operatorname{Div} \boldsymbol{\Sigma}-\boldsymbol{F}^{T} \cdot \operatorname{Div}\left\{J(\boldsymbol{J} \otimes \boldsymbol{v}) \cdot \boldsymbol{F}^{-T}\right\} .
$$

The previous implications also show the identities

$$
\boldsymbol{F}^{t} \otimes J \operatorname{div} \boldsymbol{\sigma}+\boldsymbol{F}^{t} \otimes \boldsymbol{f}_{0} \equiv \boldsymbol{F}^{t} \operatorname{Div} \boldsymbol{T}+\boldsymbol{F}^{t} \otimes \boldsymbol{f}_{0}=-\operatorname{Div} \tilde{\tilde{\boldsymbol{\Sigma}}}+\boldsymbol{F}^{t} \otimes \boldsymbol{f}_{0} .
$$

Inserting this back into the tensorial eulerian virial further delivers

$$
\begin{aligned}
\langle\hat{\boldsymbol{V}}\rangle & \equiv \int_{\boldsymbol{\Omega}_{0}} \boldsymbol{I} \cdot J \boldsymbol{\sigma}^{T} d X+\int_{\boldsymbol{\Omega}_{0}} \boldsymbol{R} \cdot\left\{\boldsymbol{F}^{T} \otimes J \operatorname{div} \boldsymbol{\sigma}+\boldsymbol{F}^{T} \otimes \boldsymbol{f}_{0}\right\} d X \\
& \equiv \int_{\boldsymbol{\Omega}_{0}} \boldsymbol{I} \cdot J \boldsymbol{\sigma}^{T} d X+\int_{\boldsymbol{\Omega}_{0}} \boldsymbol{R} \cdot\left\{-\operatorname{Div} \tilde{\tilde{\boldsymbol{\Sigma}}}+\boldsymbol{F}^{T} \otimes \boldsymbol{f}_{0}\right\} d X .
\end{aligned}
$$

Taking into account the eulerian form of the dynamical equilibrium, expressed as

$$
\nabla \cdot \sigma=\rho \frac{d \boldsymbol{v}}{d t}-\boldsymbol{f}+(\boldsymbol{J} \cdot \nabla) \boldsymbol{v}
$$

one obtains

$$
\begin{aligned}
\langle\hat{\boldsymbol{V}}\rangle \equiv & \int_{\boldsymbol{\Omega}_{0}} \boldsymbol{I} \cdot \boldsymbol{J} \boldsymbol{\sigma}^{T} d X+\int_{\boldsymbol{\Omega}_{0}} \boldsymbol{R} \cdot\left\{\boldsymbol{F}^{T} \otimes J\left\{\rho \frac{d \boldsymbol{v}}{d t}-\boldsymbol{f}+(\boldsymbol{J} \cdot \nabla) \boldsymbol{v}\right\}+\boldsymbol{F}^{T} \otimes \boldsymbol{f}_{0}\right\} d X \\
\equiv & \int_{\boldsymbol{\Omega}_{0}} \boldsymbol{I} \cdot \boldsymbol{J} \boldsymbol{\sigma}^{T} d X \\
& +\int_{\boldsymbol{\Omega}_{0}} \boldsymbol{R} \cdot\left\{-\operatorname{Div} \tilde{\boldsymbol{\Sigma}}-\boldsymbol{F}^{T} \otimes \operatorname{Div}\left\{J(\boldsymbol{J} \otimes \boldsymbol{v}) \cdot \boldsymbol{F}^{-T}\right\}+(\boldsymbol{J} \cdot \nabla) \boldsymbol{v}+\boldsymbol{F}^{T} \otimes \boldsymbol{f}_{0}\right\} d X .
\end{aligned}
$$

Further, the elaboration of Eshelby stress in terms of Cauchy stress is expressed by

$$
\boldsymbol{\Sigma}=W_{0} \boldsymbol{I}-J \boldsymbol{F}^{T} \cdot \boldsymbol{\sigma} \cdot \boldsymbol{F}^{-T}
$$

or equivalently

$$
\boldsymbol{\sigma}=-j \boldsymbol{F}^{-T} \cdot \boldsymbol{\Sigma} \cdot \boldsymbol{F}^{T}+W_{t} \boldsymbol{I},
$$


observing that the product $j W_{0}$ represents the density of strain energy in the current configuration:

$$
W_{t}:=j W_{0}
$$

This leads to a rewriting of the averaged Cauchy stress in terms of the Eshelby stress:

$$
\int_{\boldsymbol{\Omega}}\left(\boldsymbol{\sigma}^{T} \cdot \boldsymbol{I}\right) d x=\int_{\boldsymbol{\Omega}_{0}} J \boldsymbol{\sigma}^{T} \cdot \boldsymbol{I} d X=\int_{\boldsymbol{\Omega}_{0}}\left(-\boldsymbol{F}^{-T} \cdot \boldsymbol{\Sigma} \cdot \boldsymbol{F}^{T}+W_{0} \boldsymbol{I}\right) d X .
$$

Hence, the previous form of the tensorial eulerian virial becomes, after lengthy developments,

$$
\begin{aligned}
& \langle\hat{\boldsymbol{V}}\rangle \equiv \int_{\boldsymbol{\Omega}_{0}}\left(-\boldsymbol{F}^{-t} \cdot \boldsymbol{\Sigma} \cdot \boldsymbol{F}^{t}+W_{0} \boldsymbol{I}\right) d X+\int_{\boldsymbol{\Omega}_{0}} \boldsymbol{R} \cdot\left\{-\operatorname{Div} \tilde{\tilde{\boldsymbol{\Sigma}}}+\boldsymbol{F}^{t} \otimes \boldsymbol{f}_{0}\right\} d X \\
& \equiv \int_{\boldsymbol{\Omega}_{0}}\left(-\boldsymbol{F}^{-t} \cdot \boldsymbol{\Sigma} \cdot \boldsymbol{F}^{t}+W_{0} \boldsymbol{I}\right) d X, \\
& \langle\hat{\boldsymbol{V}}\rangle \equiv \int_{\boldsymbol{\Omega}_{0}} \boldsymbol{I} \cdot J \boldsymbol{\sigma}^{T} d X+\int_{\boldsymbol{\Omega}_{0}} \boldsymbol{R} \cdot\left\{\boldsymbol{F}^{T} \otimes J\left\{\rho \frac{d \boldsymbol{v}}{d t}-\boldsymbol{f}+(\boldsymbol{J} \cdot \nabla) \boldsymbol{v}\right\}+\boldsymbol{F}^{T} \otimes \boldsymbol{f}_{0}\right\} d X \\
& \equiv \int_{\boldsymbol{\Omega}_{0}}\left(-\boldsymbol{F}^{-T} \cdot \boldsymbol{\Sigma} \cdot \boldsymbol{F}^{T}+W_{0} \boldsymbol{I}\right) d X \\
& +\int_{\boldsymbol{\Omega}_{0}} \boldsymbol{R} \cdot\left\{-\operatorname{Div} \tilde{\tilde{\Sigma}}+\boldsymbol{F}^{T} \otimes f_{0}+\boldsymbol{F}^{T} \otimes J \rho \frac{d \boldsymbol{v}}{d t}\right. \\
& \left.-\boldsymbol{F}^{T} \otimes \operatorname{Div}\left\{J(\boldsymbol{J} \otimes \boldsymbol{v}) \cdot \boldsymbol{F}^{-T}\right\}+(\boldsymbol{J} . \nabla) \boldsymbol{v}\right\} d X \\
& =\int_{\boldsymbol{\Omega}_{0}}\left(-\operatorname{tr}\left(\tilde{\tilde{\Sigma}}^{t}\right)+W_{0} \boldsymbol{I}\right) d X \\
& +\int_{\boldsymbol{\Omega}_{0}} \boldsymbol{R} \cdot\left\{-\operatorname{Div} \tilde{\tilde{\Sigma}}+\boldsymbol{F}^{T} \otimes f_{0}\right. \\
& \left.-\boldsymbol{F}^{T} \otimes \operatorname{Div}\left\{J(\boldsymbol{J} \otimes \boldsymbol{v}) \cdot \boldsymbol{F}^{-T}\right\}+\boldsymbol{F}^{T} \otimes J(\boldsymbol{J} \cdot \nabla) \boldsymbol{v}\right\} d X \\
& \equiv \int_{\boldsymbol{\Omega}_{0}}\left(-\operatorname{tr}\left(\tilde{\tilde{\boldsymbol{\Sigma}}}^{t}\right)+W_{0} \boldsymbol{I}\right) d X+\int_{\boldsymbol{\Omega}_{0}} \boldsymbol{R} \cdot\left\{\boldsymbol{F}^{T} \otimes \rho J \frac{d \boldsymbol{v}}{d t}+\boldsymbol{F}^{T} \otimes J(\boldsymbol{J} \cdot \nabla) \boldsymbol{v}\right\} d X .
\end{aligned}
$$

Here we have taken into account the static equilibrium and the identity

$$
\begin{aligned}
\int_{\boldsymbol{\Omega}_{0}}\left(-\boldsymbol{F}^{-t} \cdot \boldsymbol{\Sigma} \cdot \boldsymbol{F}^{t}+W_{0} \boldsymbol{I}\right) d X & \equiv \int_{\boldsymbol{\Omega}_{0}}\left(-\boldsymbol{\Sigma}^{t}+W_{0} \boldsymbol{I}\right) d X \\
& =\int_{\boldsymbol{\Omega}_{0}}\left(-\operatorname{tr}\left(\tilde{\boldsymbol{\Sigma}}^{t}\right)+W_{0} \boldsymbol{I}\right) d X
\end{aligned}
$$

itself resulting from the equality

$$
(\boldsymbol{A} \otimes \boldsymbol{B})^{T}=\boldsymbol{B} \otimes \boldsymbol{A}, \forall \boldsymbol{A}, \boldsymbol{B},
$$


the coaxiality of $\boldsymbol{T}$ with $\boldsymbol{F}^{t}$ [Ciarlet 1993], and the following definition of the trace of a fourth-order tensor built as the dyadic product of two second-order tensors:

$$
\operatorname{tr}(\boldsymbol{A} \otimes \boldsymbol{B}):=\boldsymbol{A} \cdot \boldsymbol{B}, \forall \boldsymbol{A}, \boldsymbol{B} .
$$

According to this definition, the trace of the fourth-order Eshelby tensor in delivers the second-order Eshelby tensor.

\section{References}

[Alibert et al. 2003] J.-J. Alibert, P. Seppecher, and F. dell'Isola, "Truss modular beams with deformation energy depending on higher displacement gradients", Math. Mech. Solids 8:1 (2003), $51-73$.

[Chandrasekhar and Fermi 1953] S. Chandrasekhar and E. Fermi, "Problems of gravitational stability in the presence of a magnetic field", Astrophys. J. 118 (1953), 116-141.

[Chandrasekhar and Lebovitz 1962] S. Chandrasekhar and N. R. Lebovitz, "The potential and the superpotentials of homogeneous ellipsoids", Astrophys. J. 136 (1962), 1037-1047.

[Ciarlet 1993] P. G. Ciarlet, Mathematical elasticity: three-dimensional elasticity, vol. 1, Elsevier, North-Holland, 1993.

[Clausius 1870] R. J. E. Clausius, "On a mechanical theorem applicable to heat", Phil. Mag. (4) 40 (1870), 122-127. Reprinted as Chapter 13 (pp. 172-178) in The kinetic theory of gases, Imperial College Press, London, 2011.

[dell' Isola and Iannece 1989] F. dell'Isola and D. Iannece, "On phase transition in classical fluid mixtures with surface adsorption”, Int. J. Eng. Sci. 27:9 (1989), 1069-1078.

[dell' Isola et al. 2012] F. dell' Isola, P. Seppecher, and A. Madeo, "How contact interactions may depend on the shape of Cauchy cuts in $N$-th gradient continua: approach "à la D'Alembert"', $Z$. Angew. Math. Phys. 63:6 (2012), 1119-1141.

[dell'Isola et al. 2014] F. dell'Isola, U. Andreaus, and L. Placidi, "At the origins and in the vanguard of peridynamics, non-local and higher-gradient continuum mechanics: an underestimated and still topical contribution of Gabrio Piola”, Math. Mech. Solids 20:8 (2014), 887-928.

[Epstein and Maugin 2000] M. Epstein and G. A. Maugin, "Thermomechanics of volumetric growth in uniform bodies", Int. J. Plast. 16:7-8 (2000), 951-978.

[Eremeyev and Pietraszkiewicz 2009] V. A. Eremeyev and W. Pietraszkiewicz, "Phase transitions in thermoelastic and thermoviscoelastic shells", Arch. Mech. Stos. 61:1 (2009), 41-67.

[Eremeyev and Pietraszkiewicz 2011] V. A. Eremeyev and W. Pietraszkiewicz, "Thermomechanics of shells undergoing phase transition”, J. Mech. Phys. Solids 59:7 (2011), 1395-1412.

[Ericksen 1984] J. L. Ericksen, "The Cauchy and Born hypotheses for crystals", pp. 61-77 in Phase transformations and material instabilities in solids (Madison, Wis., 1983), Publ. Math. Res. Center Univ. Wisconsin 52, Academic Press, Orlando, FL, 1984.

[Ganghoffer 2010a] J.-F. Ganghoffer, "On Eshelby tensors in the context of the thermodynamics of open systems: application to volumetric growth", Int. J. Eng. Sci. 48:12 (2010), 2081-2098.

[Ganghoffer 2010b] J.-F. Ganghoffer, "On the generalized virial theorem and Eshelby tensors", Int. J. Solids Struct. 47:9 (2010), 1209-1220.

[Ganghoffer 2012] J. F. Ganghoffer, "Extremum principles for biological continuous bodies undergoing volumetric and surface growth”, Bull. Polish Acad. Sci. Tech. Sci. 60:2 (2012), 259. 
[Gommerstadt 2001] B. Gommerstadt, "M-integral and virial theorem in elastodynamics", Int. J. Fract. 112:3 (2001), 33-38.

[Irving and Kirkwood 1950] J. H. Irving and J. G. Kirkwood, "The statistical mechanical theory of transport processes. IV. The equations of hydrodynamics”, J. Chem. Phys. 18 (1950), 817-829.

[Jouanna and Brocas 2001] P. Jouanna and S. Brocas, "Approche statistique du tenseur des contraintes par le viriel généralisé”, C. R. Acad. Sci. Paris Ser. IIB Mech. 329:11 (2001), 775-782.

[Jouanna and Pèdesseau 2004] P. Jouanna and L. Pèdesseau, "Partial stresses in heterogeneous media by a direct statistical approach", C. R. Mécanique 332:4 (2004), 305-312.

[Maugin 1993] G. A. Maugin, Material inhomogeneities in elasticity, Applied Mathematics and Mathematical Computation 3, Chapman \& Hall, London, 1993.

[Milstein 1982] F. Milstein, "Crystal elasticity”, pp. 417-452 in Mechanics of solids, edited by H. G. Hopkins and M. J. Sewell, Pergamon, Oxford, 1982.

[Misra and Poorsolhjouy 2015a] A. Misra and P. Poorsolhjouy, "Granular micromechanics based micromorphic model predicts frequency band gaps”, Contin. Mech. Therm. (2015), 1-20.

[Misra and Poorsolhjouy 2015b] A. Misra and P. Poorsolhjouy, "Granular micromechanics model for damage and plasticity of cementitious materials based upon thermomechanics", Math. Mech. Solids (published online March 2015).

[Misra and Singh 2015] A. Misra and V. Singh, "Thermomechanics-based nonlinear rate-dependent coupled damage-plasticity granular micromechanics model”, Contin. Mech. Therm. 27:4-5 (2015), 787-817.

[Murdoch 2007] A. I. Murdoch, "A critique of atomistic definitions of the stress tensor", J. Elasticity 88:2 (2007), 113-140.

[Parker 1954] E. N. Parker, "Tensor virial equations", Phys. Rev. (2) 96:6 (1954), 1686-1689.

[Steinmann 2000] P. Steinmann, "Application of material forces to hyperelastostatic fracture mechanics, I: Continuum mechanical setting", Int. J. Solids Struct. 37:48-50 (2000), 7371-7391.

[Sunyk and Steinmann 2003] R. Sunyk and P. Steinmann, "On higher gradients in continuum-atomistic modelling”, Int. J. Solids Struct. 40:24 (2003), 6877-6896.

[Tadmor et al. 1996] E. B. Tadmor, M. Ortiz, and R. Phillips, "Quasicontinuum analysis of defects in solids", Philos. Mag. A 73:6 (1996), 1529-1563.

Received 2 May 2015. Revised 26 Aug 2015. Accepted 19 Oct 2015.

JEAN-FRANÇOIS GANGHOFFER: jean-francois.Ganghoffer@univ-lorraine.fr

LEMTA, Université de Lorraine, 2, Avenue de la Forêt de Haye, TSA 60604, 54504 Vandoeuvre, France 
EDITORIAL BOARD

ANTONIO CARCATERRA

ERIC A. CARLEN

FRANCESCO DELL'ISOLA

RAFFAELE ESPOSITO

ALBERT FANNJIANG

Gilles A. FranCFORT

Pierangelo MARCATI

JEAN-JACQUES MARIGO

PETER A. MARKOWICH

MARTIN OSTOJA-STARZEWSKI

PIERRE SEPPECHER

DAVID J. STEIGMANN

PAUl STEINMANN

PierRe M. SuQueT

MANAGING EDITORS

MICOL AMAR

CORRADO LATTANZIO

ANGELA MADEO

MARTIN OSTOJA-STARZEWSKI

ADVISORY BOARD

ADNAN AKAY

Holm AltenBaCH

MICOL AMAR

HARM ASKES

TEODOR ATANACKOVIĆ

VICTOR BERDICHEVSKY

GUY BOUCHITTÉ

ANDREA BRAIDES

ROBERTO CAMASSA

MAURO CARFORE

ERIC DARVE

FELIX DARVE

ANNA DE MASI

GianPiEtro DEL Piero

EMMANUELE Di BENEDETTO

BERNOLD FIEDLER

IRENE M. GAMBA

DAVID Y. GAO

SERGEY GAVRILYUK

TIMOTHY J. HEALEY

DOMINIQUE JEULIN

ROGER E. KHAYAT

CORRADO LATTANZIO

ROBERT P. LIPTON

ANGELO LUONGO

ANGELA MADEO

JUAN J. MANFREDI

CARLO MARCHIORO

GÉRARD A. MAUGIN

ROBERTO NATALINI PATRIZIO NEFF

ANDREY PIATNITSKI

ERRICO PRESUTTI

MARIO PULVIRENTI

LUCIO RUSSO

Miguel A. F. SANJUAN

PATRICK SElVADURAI

ALEXANDER P. SEYRANIAN

MIROSLAV ŠILHAVÝ

GUIDO SWEERS

ANTOINETTE TORDESILLAS

LEV TRUSKINOVSKY

JUAN J. L. VELÁZQUEZ VINCENZO VESPRI ANGELO VULPIANI msp.org/memocs

Università di Roma "La Sapienza", Italia

Rutgers University, USA

(CO-CHAIR) Università di Roma "La Sapienza", Italia

(TREASURER) Università dell'Aquila, Italia

University of California at Davis, USA

(CO-CHAIR) Université Paris-Nord, France

Università dell'Aquila, Italy

École Polytechnique, France

DAMTP Cambridge, UK, and University of Vienna, Austria

(CHAIR MANAGING EDITOR) Univ. of Illinois at Urbana-Champaign, USA

Université du Sud Toulon-Var, France

University of California at Berkeley, USA

Universität Erlangen-Nürnberg, Germany

LMA CNRS Marseille, France

Università di Roma "La Sapienza", Italia

Università dell'Aquila, Italy

Université de Lyon-INSA (Institut National des Sciences Appliquées), France

(CHAIR MANAGING EDITOR) Univ. of Illinois at Urbana-Champaign, USA

Carnegie Mellon University, USA, and Bilkent University, Turkey

Otto-von-Guericke-Universität Magdeburg, Germany

Università di Roma "La Sapienza", Italia

University of Sheffield, UK

University of Novi Sad, Serbia

Wayne State University, USA

Université du Sud Toulon-Var, France

Università di Roma Tor Vergata, Italia

University of North Carolina at Chapel Hill, USA

Università di Pavia, Italia

Stanford University, USA

Institut Polytechnique de Grenoble, France

Università dell'Aquila, Italia

Università di Ferrara and International Research Center MEMOCS, Italia

Vanderbilt University, USA

Freie Universität Berlin, Germany

University of Texas at Austin, USA

Federation University and Australian National University, Australia

Université Aix-Marseille, France

Cornell University, USA

École des Mines, France

University of Western Ontario, Canada

Università dell' Aquila, Italy

Louisiana State University, USA

Università dell'Aquila, Italia

Université de Lyon-INSA (Institut National des Sciences Appliquées), France University of Pittsburgh, USA

Università di Roma "La Sapienza”, Italia

Université Paris VI, France

Istituto per le Applicazioni del Calcolo "M. Picone", Italy

Universität Duisburg-Essen, Germany

Narvik University College, Norway, Russia

Università di Roma Tor Vergata, Italy

Università di Roma "La Sapienza”, Italia

Università di Roma “Tor Vergata”, Italia

Universidad Rey Juan Carlos, Madrid, Spain

McGill University, Canada

Moscow State Lomonosov University, Russia

Academy of Sciences of the Czech Republic

Universität zu Köln, Germany

University of Melbourne, Australia

École Polytechnique, France

Bonn University, Germany

Università di Firenze, Italia

Università di Roma La Sapienza, Italia

MEMOCS (ISSN 2325-3444 electronic, 2326-7186 printed) is a journal of the International Research Center for the Mathematics and Mechanics of Complex Systems at the Università dell'Aquila, Italy.

Cover image: "Tangle” by $\odot$ John Horigan; produced using the Context Free program (contextfreeart.org).

PUBLISHED BY

7 mathematical sciences publishers

nonprofit scientific publishing

http://msp.org/

(C) 2015 Mathematical Sciences Publishers 
Mathematics and Mechanics of Complex Systems vol. 3 no. 4

An analysis of the latitudinal data of Eratosthenes and Hipparchus

\section{Christian Marx}

Spatial and material stress tensors in continuum mechanics 341 of growing solid bodies

Jean-François Ganghoffer

A crack with surface elasticity in finite plane elastostatics 365 $\mathrm{Xu}$ Wang and Peter Schiavone

An investigation of the active damping of suspension bridges

André Preumont, Matteo Voltan, Andrea Sangiovanni, Renaud Bastaits, Bilal Mokrani and David Alaluf

MEMOCS is a journal of the International Research Center for the Mathematics and Mechanics of Complex Systems at the Università dell' Aquila, Italy.

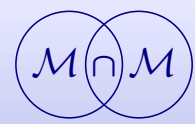

